\title{
OPEN Photoreceptor alteration in intermediate age-related macular degeneration
}

\author{
Enrico Borrelli@1,2, Riccardo Sacconi ${ }^{1,2}$, Biancamaria Zuccaro ${ }^{2}$, Michele Cavalleri ${ }^{1,2}$, \\ Alessandro Bordato ${ }^{1,2}$, Ilaria Zucchiatti ${ }^{1,2}$, Lea Querques ${ }^{1,2}$, Francesco Bandello ${ }^{1,2}$ \& \\ Giuseppe Querques $\mathbb{1}^{1,2 \bowtie}$
}

The aim of this study was to analyze photoreceptor alterations occurring in eyes with intermediate age-related macular degeneration (AMD) and to investigate their associations with choriocapillaris (CC) flow. In this retrospective case-control study, we collected data from 35 eyes with intermediate AMD from 35 patients who had swept source optical coherence tomography structural and angiography imaging obtained. A control group of 35 eyes from 35 healthy subjects was included for comparison. Our main outcome measure for comparison between groups was the normalized reflectivity of en face image segmented at the ellipsoid zone (EZ) level, which was calculated to quantify the photoreceptor damage. OCTA metrics to quantify CC flow signal were also computed. These metrics were measured in a circle centered on the fovea and with a diameter of $5 \mathrm{~mm}$. In intermediate AMD eyes, the macular area occupied by drusen was identified. Therefore, the EZ reflectivity and CC flow signal were separately measured in regions without drusen ("drusen-free" region). Measurements were generated using previously published algorithms. Mean \pm SD age was $74.1 \pm 6.8$ years in the intermediate AMD group and $72.1 \pm 6.0$ years in the control group $(p=0.206)$. The normalized EZ reflectivity was $0.76 \pm 0.10$ in the intermediate AMD group and $0.85 \pm 0.08$ in the control group $(p<.0001)$. In the "drusen-free" region, the normalized EZ reflectivity was $0.77 \pm 0.10$ $(p<.0001$ vs. healthy controls) and was positively correlated with the CC flow signal density $(\rho=-0.340$ and $p=0.020)$. In conclusion, eyes with intermediate AMD exhibit a diffuse reduced EZ normalized reflectivity, and this reduction is correlated with CC flow signal in the regions without drusen. This study supports the concept of the damage of the unit comprised of photoreceptor, CC, and intervening tissues as an early event in AMD.

Age-related macular degeneration (AMD) is a major cause of decreased vision among elderly individuals in developed countries ${ }^{1}$. This disorder may present at different stages and the "intermediate AMD" stage is clinically featured by the presence of pigmentary abnormalities and/or large drusen ${ }^{2}$.

Although the AMD pathogenesis is intricate and related with many systemic and lifestyle factors that may have a role in the development and progression of this disorder ${ }^{3,4}$, a growing evidence suggests that this disorder is ultimately characterized by damage of the unit comprised of photoreceptors, retinal pigment epithelium (RPE), Bruch's membrane, and choriocapillaris (CC $)^{5-7}$. Importantly, several evidences suggest that this may be considered as a tightly knit, integrated unit ${ }^{8-10}$. In AMD, this impairment causes the development of drusen and progressive photoreceptor, RPE and CC degeneration ${ }^{11-13}$.

Although photoreceptor loss is associated with aging ${ }^{14}$, both histopathological and imaging studies have displayed that intermediate AMD eyes may be characterized by a greater photoreceptor loss ${ }^{15,16}$. Alterations in CC flow are also associated with aging ${ }^{13,17-19}$ and a greater reduction in CC flow was demonstrated to occur in intermediate AMD eyes ${ }^{20-25}$. Therefore, a greater reduction in CC flow in these eyes might provide a potential rationale for a damage in photoreceptors via an ischemic mechanism ${ }^{11}$.

En face optical coherence tomography (OCT) imaging provides a topographic qualitative and quantitative assessment of photoreceptors. On commercial structural OCT, the ellipsoid zone (EZ) layer (formerly known as inner segment/outer segment junction) is characterized by an high reflectivity attributed to numerous mitochondria in photoreceptor inner segment ellipsoids ${ }^{26}$. On the en face structural OCT images, anomalies in the EZ 
may be displayed as hyporeflective regions. Therefore, the EZ reflectivity has been employed in several previous reports as a readout of photoreceptor damage ${ }^{16,27-29}$.

In the present study, we provided a quantitative assessment of the photoreceptor damage in intermediate AMD by employing a standardized methodology ${ }^{16,29}$ to objectively measure the EZ reflectivity. Our aim was to explore the relationship between photoreceptor damage and CC flow signal in these eyes. Measurements were performed in a circular region of interest (ROI) centered on the fovea and with a diameter of $5 \mathrm{~mm}$. Furthermore, our analyses were also separately assessed in areas without drusen ("drusen-free" region). The latter choice was consequent to the following reasons: (i) the measurements of the EZ may be altered in regions with drusen because of the occurrence of EZ distortion and/or segmentation errors; (i) the quantification of CC flow signal may be more affected by shadowing artifacts in regions colocalizing with drusen; and (iii) photoreceptors may be regionally affected by drusen through a mechanical compression or by impeding the transition of oxygen and nutrients from the CC to the outer retina. Therefore, the assessment of associations between EZ reflectivity and CC flow signal in regions without drusen was aimed at understanding the relationship between these two variables after restricting the above-mentioned confounding factors. Our findings could be helpful to better comprehend the AMD pathophysiology, and to recognize new targets for therapy.

\section{Methods}

Study participants. This study was a retrospective case-control analysis. The authors in this study identified patients with a clinical diagnosis of intermediate AMD in at least one eye as determined by clinical examination and by structural $\mathrm{OCT}^{2}$. All patients were imaged with the PLEX Elite 9000 device (Carl Zeiss Meditec Inc., Dublin, CA, USA) between July 2018 and October 2019. Written informed consent was obtained from all subjects, and it was approved by the San Raffaele Ethics Committee. The study adhered to the tenets of the Declaration of Helsinki and Health Insurance Portability and Accountability Act.

Exclusion criteria for intermediate AMD eyes were: (i) history of ocular surgery or anti-vascular endothelial growth factor (VEGF) injection; (ii) myopia greater than - 3.00 diopters; (iii) presence of subretinal drusenoid deposits (i.e. reticular pseudodrusen) as determined with OCT analysis ${ }^{30}$, since its presence may alter the assessment of the reflectivity of the en face EZ image; (iv) presence of type 1 non-exudative quiescent neovascularization as determined with OCT angiography (OCTA) analysis ${ }^{31}$; and (v) any maculopathy secondary to causes other than AMD.

Because age may influence quantitative measurements of the CC on OCTA ${ }^{18,32}$, a control group similar with respect to age and gender was also included in the current analysis. All control subjects failed to demonstrate evidence of ocular disease or media opacity as evaluated by dilated fundus examination, OCT, and OCTA analysis.

Furthermore, we excluded poor quality images with a signal strength index lower than 7 (a measurement in a scale $0-10$ indicating the level of retinal tissue signal with respect to the noise or background level in OCT data), as recommended by manufacturers and applied in previous studies ${ }^{18,33}$.

Imaging. Patients underwent SS-OCT and SS-OCTA imaging using the PLEX Elite 9000 device (Carl Zeiss Meditec Inc., Dublin, CA, USA) which uses a swept laser source with a central wavelength of $1050 \mathrm{~nm}(1000-$ $1100 \mathrm{~nm}$ full bandwidth) and operating at 100,000 A-scans per second. This instrument employs a full-width at half-maximum (FWHM) axial resolution of approximately $5 \mu \mathrm{m}$ in tissue, and a lateral resolution at the retinal surface estimated at approximately $14 \mu \mathrm{m}$. Structural OCT and OCTA imaging of the macula included a $6 \times 6-\mathrm{mm}$ field of view area centered on the fovea (500 A-scans $\times 500 \mathrm{~B}$-scans).

Image processing. Delineation of the region without drusen. In order to identify the region without drusen ("drusen-free" region), we used the advanced RPE analysis version 0.4 algorithm which is available on the ARI network by Zeiss (https://arinetworkhub.com). This algorithm is aimed at generating the RPE elevation map with respect to Bruch's membrane. The RPE elevation en face map is a ".png" image describing the degree of RPE elevation in an en face map. In details, different colors indicate the degree of elevation in the form of a heat-map, from $0 \mu \mathrm{m}$ elevation (in blue) to a maximum of $100 \mu \mathrm{m}$ elevation (in bright red) (Figs. 1, 2, 3). The algorithm also outputs quantitative data indicating the en face area of the RPE that is considerably elevated from Bruch's membrane (drusen area), as well as the volume of such elevation (drusen volume), in a circle centered on the fovea and with a diameter of $5 \mathrm{~mm}$. The position of the fovea was selected manually using the structural OCT. In details, the foveal center was manually annotated on the CC and EZ en face images by using the structural OCT volume as reference.

The en face elevation map image was imported into ImageJ, and the "MaxEntropy" threshold was applied to "binarize" this image. This binarization threshold was previously used to binarize an elevation map image in intermediate AMD eyes ${ }^{21}$ and also in the present study was evaluated as an effective algorithm to select the drusen region, as evaluated by two experienced graders (EB and RS). In the binarized image, the "drusen" and "drusen-free" regions were displayed with two different colors (Fig. 1).

Quantification of the EZ normalized reflectivity. The EZ normalized reflectivity is a dimensionless metric that was measured as previously shown (Fig. 1) ${ }^{16,29}$. In brief, the structural scan of the macula was used to automatically generate the EZ en face image-slab of $21 \mu \mathrm{m}$ with the inner boundary placed $45 \mu \mathrm{m}$ above the RPE reference, as previously described ${ }^{16}$. This image was imported into Fiji ImageJ software version 2.0.0 (National Institutes of Health, Bethesda, MD; available at http://rsb.info.nih.gov/ij/index.html). The mean brightness of the EZ en face image was thus measured in a circular region of interest centered on the fovea and with a diameter of $5.0 \mathrm{~mm}$. Assuming that the assessment of the EZ may be altered in regions with drusen because of the occurrence of EZ distortion and/or segmentation errors, this measurement was also separately performed in 

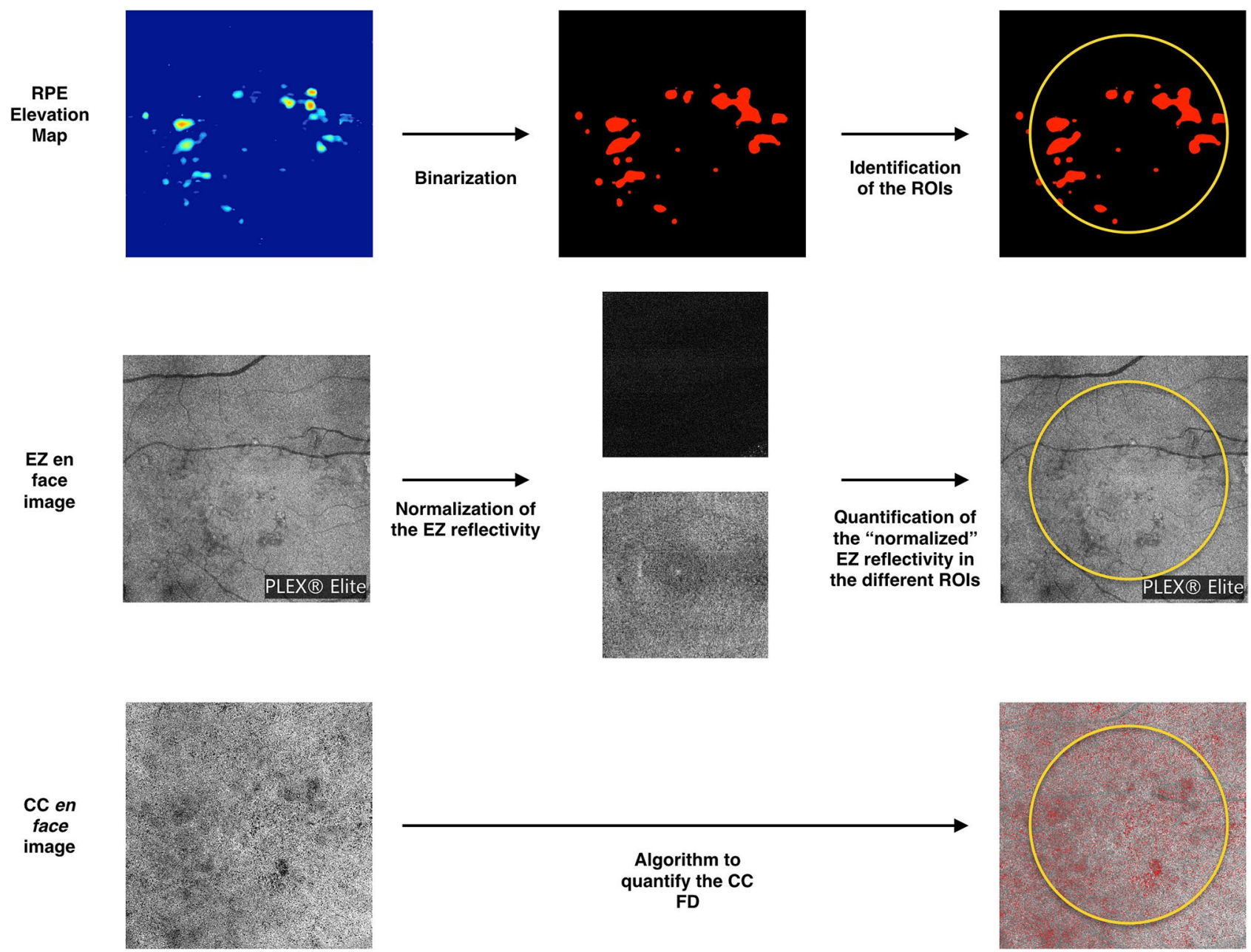

Figure 1. Representation of the algorithm used to investigate the images. The en face RPE elevation map image was used to identify regions occupied by drusen. This image was imported into ImageJ, and the "MaxEntropy" threshold was applied to "binarize" this image. In the binarized image, the "drusen" region (red) was excluded in a following analysis which was aimed at measuring metrics in the "drusen-free" region. Measurements were performed in a circular region of interest centered on the fovea (diameter of $5.0 \mathrm{~mm}$-displayed in yellow in the figure). The en face image of the ellipsoid zone (EZ) was also imported in ImageJ. A previously described imageprocessing algorithm using two reference structures, the vitreous and the retinal nerve fiber layer, was employed to calculate the EZ normalized reflectivity. The en face flow image of the CC was compensated using the corresponding CC en face structural image. A local thresholding method was applied in order to detect pixels falling below this threshold (in red in the lower right image). Values detected in this example are, as follows: drusen area $=1.36 \mathrm{~mm}^{2}$; drusen volume $=0.05 \mathrm{~mm}^{3}$; normalized EZ reflectivity in the ROI $=0.79$; normalized EZ reflectivity in the drusen-free region $=0.80$; CC FD\% $=24.2 \%$; CC FD $\%$ in the drusen-free region $=24.1 \%$.

the "drusen-free" regions contained in this circular region of interest. Of note, this measurement was made after excluding the region occupied by major retinal vessels, as previously showed ${ }^{29}$.

Since the brightness of structures displayed using structural OCT may depend on a variety of uncontrollable factors, we employed a previously described image-processing algorithm to normalize the signal across a cohort $^{16,29,34-36}$. For each eye, this algorithm uses the vitreous and the retinal nerve fiber layer (RNFL) as dark and bright references, respectively. After testing the mean brightness in these structures, a formula was applied to obtain the EZ normalized reflectivity for each eye and analyzed region. As the inter-observer agreement for EZ normalized reflectivity algorithm has been reported previously and demonstrated to be excellent in intermediate AMD and control eyes ${ }^{16}$, this was not reassessed in the present study (Figs. 1, 2, 3).

Measurement of the CC flow signal. In order to quantify the CC flow signal, we used the multilayer segmentation algorithm (version 0.5) which is available on the ARI network by Zeiss (https://arinetworkhub.com). This algorithm consists on a graph-based approach using a combination of intensity, axial gradient, gradient magnitude and positional information as cost functions to find the optimal location of each layer within a B-scan or several adjacent B-scans. The en face OCTA images of the CC were generated using a $20 \mu \mathrm{m}$ thick slab that followed the contour of the RPE fit boundary, where Bruch's membrane is usually located, and was placed at about $29 \mu \mathrm{m}$ under this structure, as set by the manufacturer ${ }^{37,38}$. Importantly, using this algorithm, the RPE fit 

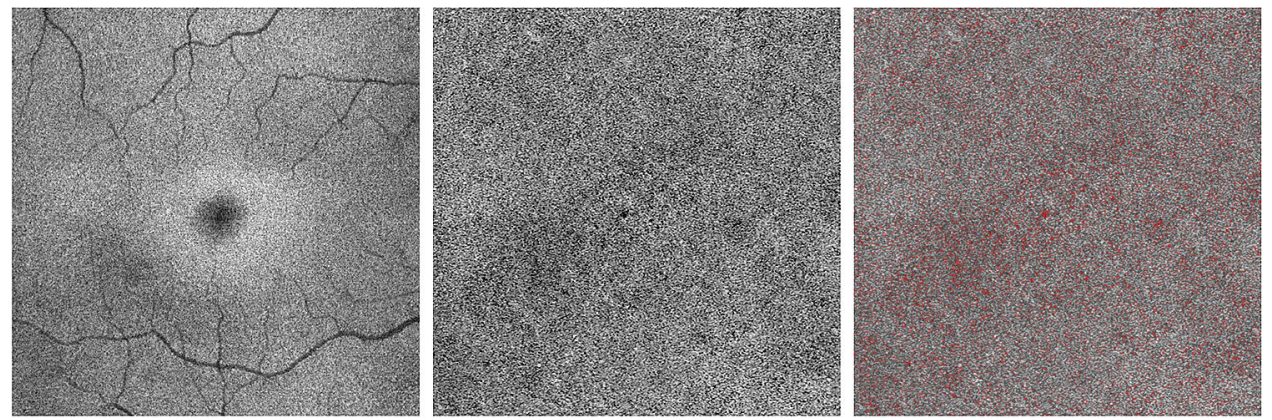

Figure 2. Representative images from an healthy control eye. The en face EZ image (middle-left) is obtained with the slab displayed in the reference OCT B-scan image (left). The en face EZ image demonstrates a uniform reflectivity which may indicate a normal photoreceptor structure. The original (middle-right) and postprocessing (right) CC OCTA images show areas of flow deficits (in red in the right image) which are more localized in the foveal region.
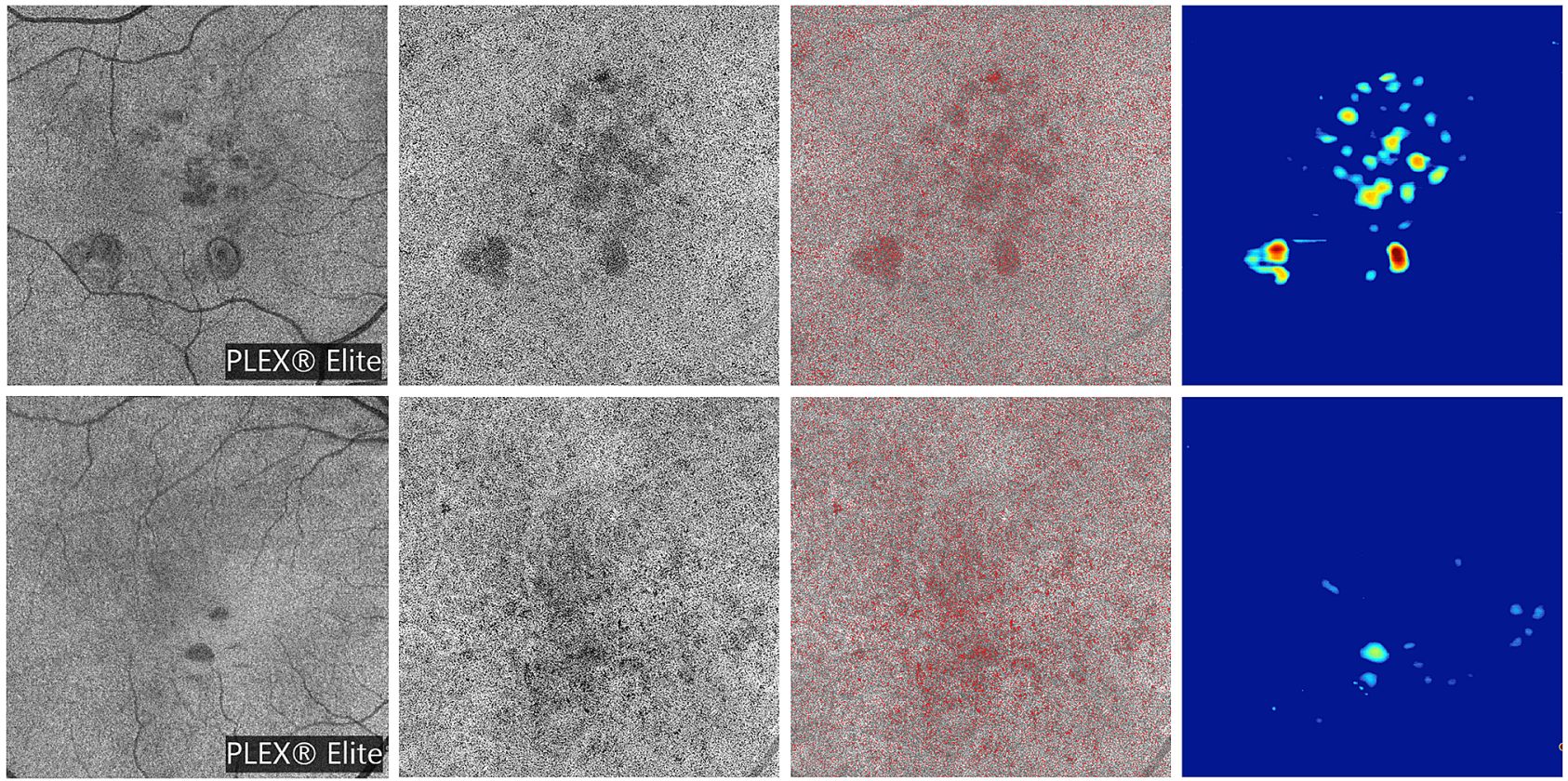

Figure 3. Representative images from intermediate AMD eyes. The OCT B-scan images (first column) show the slab set to visualize the EZ image. The en face EZ images (second column) display several regions of reduced reflectivity, this suggesting the presence of areas of photoreceptor impairment that seems to be greater in areas with drusen. The original (third column) and post-processing (fourth column) CC images show areas of increased FD percentage, which are mainly located in the regions occupied by drusen. The en face RPE elevation map images (fifth column) display the distribution of drusen in the macular region.

boundary does not represent a direct segmentation of Bruch's membrane generated directly from the OCT data, but it is actually a surface fit to the RPE fit boundary excluding any region of pathology.

The obtained image was compensated using the corresponding CC en face structural image in order to adjust for shadowing artifacts ${ }^{22,39,40}$ and retinal vessel projection artifacts were therefore removed. The Phansalkar method was used to binarize the images, as previously described $22,32,41-43$. As recently suggested, the Phansalkar threshold was set with a window radius of 3 pixels $\left(\sim 103 \mu \mathrm{m}^{2}\right)$, as recently suggested by Chu and colleagues ${ }^{44}$. In the latter study, the authors proved that the latter radius is the best strategy for analyzing $6 \times 6-\mathrm{mm}$ scans with a $1024 \times 1024$ size. Finally, as suggested by Chu et al. ${ }^{44}$, the isolated regions with an equivalent diameter smaller than $24 \mu \mathrm{m}$ were subsequently removed from the obtained binarized images, as they were presumed to represent noise $^{45}$. Recent histopathological evidences have questioned this choice as high-quality histomorphometry has demonstrated that many spacings in the CC are even smaller that $24 \mu \mathrm{m}^{46}$. However, we felt the safest strategy was to employ the algorithm by Chu et al. ${ }^{44}$ that has been validated in the assessment of the CC flow signal. 


\begin{tabular}{|c|c|c|c|}
\hline & \multirow[b]{2}{*}{ Controls } & \multicolumn{2}{|l|}{ Intermediate AMD } \\
\hline & & Whole region & Drusen-free region \\
\hline \multirow{2}{*}{ EZ normalized reflectivity } & \multirow{2}{*}{$\begin{array}{l}0.85 \pm 0.08 \\
0.86(0.79-0.92)\end{array}$} & $\begin{array}{l}0.76 \pm 0.10 \\
0.76(0.71-0.84)\end{array}$ & $\begin{array}{l}0.77 \pm 0.10 \\
0.76(0.71-0.84)\end{array}$ \\
\hline & & $<.0001^{\mathrm{a}}$ & $<.0001^{\mathrm{a}}$ \\
\hline \multirow{2}{*}{ Choriocapillaris FD\% } & \multirow{2}{*}{$\begin{array}{l}19.2 \pm 6.2 \\
20.7(13.9-23.5)\end{array}$} & $\begin{array}{l}24.1 \pm 5.3 \\
23.9(21.9-28.1) \\
\end{array}$ & $\begin{array}{l}24.0 \pm 5.4 \\
23.9(21.9-28.1) \\
\end{array}$ \\
\hline & & $0.001^{\mathrm{a}}$ & $0.001^{\mathrm{a}}$ \\
\hline \multirow{2}{*}{ Choriocapillaris FD average size $\left(\mu \mathrm{m}^{2}\right)$} & \multirow{2}{*}{$\begin{array}{l}612.0 \pm 72.6612 .0 \pm 72.6 \\
590.4(576.4-639.6)\end{array}$} & $\begin{array}{l}898.2 \pm 289.6898 .2 \pm 289.6 \\
822.2(690.8-943.4)\end{array}$ & $\begin{array}{l}747.0 \pm 151.0 \\
748.6(639.6-832.4)\end{array}$ \\
\hline & & $<.0001^{\mathrm{b}}$ & $<.0001^{\mathrm{b}}$ \\
\hline
\end{tabular}

Table 1. Tested variables in controls and age-related macular degeneration patients. Data are presented as mean \pm SD (standard deviation) and median (interquartile range). AMD: age-related macular degeneration; EZ: ellipsoid zone; FD\%: percentage of flow deficits. ${ }^{a}$ Independent samples T test-comparison with controls. ${ }^{\mathrm{b}}$ Independent samples Mann-Whitney U test-comparison with controls.

The final binarized image was thus imported in Fiji ImageJ software version 2.0.0 (National Institutes of Health, Bethesda, MD; available at http://rsb.info.nih.gov/ij/index.html) and processed with the 'Analyze Particles' command, in order to count and measure the flow signal voids in a circular region of interest centered on the fovea (diameter of $5.0 \mathrm{~mm}$ ). The percentage of flow deficits (FD\%) and the average size of the flow deficits were calculated. As also the CC assessment may be significantly affected in regions with drusen because of shadowing artifacts, in the central $5 \mathrm{~mm}$ diameter of intermediate AMD eyes, the CC measurements were also separately performed in the "drusen-free" region (Figs. 1, 2, 3).

Statistical analysis. All quantitative variables were reported as mean and standard deviation (SD) and/or median and interquartile range (IQR) in the Results section and in Table 1.

To detect deviations from a normal distribution, Shapiro-Wilk's test was performed for all variables. Independent samples T test and non-parametric Mann Whitney $U$ test were conducted to investigate differences in continuous variables between the AMD and control groups.

Spearman's correlation coefficient was used to assess correlations between EZ normalized reflectivity and other variables.

Statistical calculations were performed using Statistical Package for Social Sciences (version 20.0, SPSS Inc., Chicago, IL, USA). The chosen level of statistical significance was $\mathrm{p}<0.05$.

The sample size of the study was tested to be proper for a mean difference between groups of almost $10 \%$, a power of $80 \%$ and type I error rate $(\alpha)$ of $5 \%$.

\section{Results}

Characteristics of patients included in the analysis. Of the 70 patients (70 eyes) included in this analysis, 35 ( 21 female) were diagnosed with intermediate AMD and 35 ( 22 female) were healthy controls. In the intermediate AMD group, 27 patients presented with bilateral intermediate AMD, whereas 8 patients presented with neovascular AMD in the fellow eye. Mean \pm SD age was $74.1 \pm 6.8$ years [range $64-88$ years] in the intermediate AMD group and $72.1 \pm 6.0$ years [range $62-85$ years] in the control group $(\mathrm{p}=0.206)$.

In intermediate AMD eyes, the drusen area was $0.72 \pm 0.73 \mathrm{~mm}^{2}$ [median $=0.44 \mathrm{~mm}^{2} ; \mathrm{IQR}=0.1-1.34 \mathrm{~mm}^{2}$ ], while the drusen volume was $0.026 \pm 0.028 \mathrm{~mm}^{3}$ [median $=0.012 \mathrm{~mm}^{3}$; IQR $\left.=0.003-0.049 \mathrm{~mm}^{3}\right]$, as evaluated using the advanced RPE analysis software. The percent of area occupied by drusen in the central $5 \mathrm{~mm}$ diameter was $3.8 \pm 3.2 \%$.

Comparison between the two groups. The normalized EZ reflectivity was $0.76 \pm 0.10$ [median $=0.76$; $\mathrm{IQR}=0.71-0.84$ ] in the intermediate AMD group and $0.85 \pm 0.08$ [median $=0.86 ; \mathrm{IQR}=0.79-0.92$ ] in the control group $(\mathrm{p}<0.0001)$. As specified above, assuming that the assessment of the EZ reflectivity and CC flow signal may be challenging in the "drusen" region, we performed a separate analysis considering only the "drusen-free" region. In this analysis, the normalized EZ reflectivity was $0.77 \pm 0.10$ [median $=0.76 ; \mathrm{IQR}=0.71-0.84$ ] in the "drusen-free" region ( $\mathrm{p}<0.0001$ vs healthy controls).

The CC FD\% was significantly increased in intermediate AMD eyes compared to control eyes $(24.1 \pm 5.3 \%$, median $=23.9 \%$ and IQR $21.9-28.1 \%$ in the intermediate AMD group; $19.2 \pm 6.2 \%$, median $=20.7 \%$ and IQR $13.9-23.5 \%$ in the control group; $\mathrm{p}=0.001)$. Similar differences were detected in the "drusen-free" region (Table 1).

The average size of the CC flow signal deficits was increased in intermediate AMD eyes $\left(898.2 \pm 289.6 \mu \mathrm{m}^{2}\right.$, median: $822.2 \mu \mathrm{m}^{2}$ and IQR: $690.8-943.4 \mu \mathrm{m}^{2}$ in the intermediate AMD group; $612.0 \pm 72.6 \mu \mathrm{m}^{2}$, median: 590.4 $\mu \mathrm{m}^{2}$ and IQR: $576.4-639.6 \mu \mathrm{m}^{2}$ in the normal group; $\left.\mathrm{p}<0.0001\right)$. The average FD size was significantly increased also considering the region without drusen $\left(747.0 \pm 151.0 \mu \mathrm{m}^{2}\right.$, median: $748.6 \mu \mathrm{m}^{2}$ and IQR: $\left.639.6-832.4 \mu \mathrm{m}^{2}\right)$, as compared with control eyes $(\mathrm{p}<0.0001$ ) (Table 1$)$. The CC FD\% and EZ normalized reflectivity were $26.5 \pm 6.8 \%$ and $0.71 \pm 0.11$ in the regions with drusen, respectively. 
Correlation analysis. Considering the whole 5-mm-diameter region of interest (ROI), the EZ normalized reflectivity was inversely correlated with the drusen area $(\rho=-0.371$ and $p=0.027)$ and volume $(\rho=-0.388$ and $p=0.021)$, while it was not associated with both the CC FD percentage $(\rho=0.257$ and $p=0.102)$ and average size $(\rho=-0.088$ and $p=0.715)$. In the analysis considering only the "drusen-free" region, the EZ normalized reflectivity was inversely correlated with the CC FD\% $(\rho=-0.340$ and $p=0.020)$, but was not correlated with average FD size $(\rho=0.050$ and $p=0.765)$.

In control eyes, the EZ normalized reflectivity was not associated with the CC variables $(\rho=-0.075$ and $\mathrm{p}=0.668$ for $\mathrm{FD} \%$ and $\rho=0.007$ and $\mathrm{p}=0.969$ for FD average size).

\section{Discussion}

In this retrospective case-control study we investigated the EZ reflectivity in intermediate AMD and healthy eyes. Our results showed that photoreceptors are affected in intermediate AMD eyes. Importantly, the EZ reflectivity was also reduced in areas without drusen, which may suggest that these eyes experience an early photoreceptor alteration impacting reflectivity. Furthermore, we also demonstrated a moderate relationship between CC flow signal and EZ normalized reflectivity in the "drusen-free" region of intermediate AMD eyes, whereas control eyes did not display a similar association. Hence, our findings may imply a pathological and early connection between CC and photoreceptors in these eyes.

Data from a number of studies using distinct approaches indicate that photoreceptors may be affected in AMD eyes ${ }^{15,47}$. Histopathological studies have demonstrated that photoreceptor alterations increase in presence of AMD. Curcio and colleagues ${ }^{15}$ have extensively studied the quantity of photoreceptors in eyes with mid- to late-stage AMD. They analyzed postmortem eyes from 7 donors ( 13 eyes) with AMD, including 5 nonexudative AMD eyes, and their findings were compared with age-matched controls. They observed that eyes with drusen were characterized by a significant reduction in photoreceptor number and that this decrease was mainly confined to the parafovea ${ }^{15}$. Importantly, there was heterogenicity in photoreceptor loss among donors, as in two donors, rod loss exceeded cone loss at most parafoveal locations, and in one donor, rod density was normal and cone density was reduced ${ }^{15}$. Using adaptive optics scanning laser ophthalmoscope, Boretsky et al. ${ }^{47}$ investigated 4 patients with AMD (one of these patients was affected by intermediate AMD). In the latter study, the authors demonstrated a progressive reduction in photoreceptors' density throughout the successive AMD stages $^{47}$. Furthermore, using structural OCT, Rogala and colleagues ${ }^{48}$ illustrated an extensive outer retinal thinning in intermediate AMD eyes, as compared with age-matched controls. Finally, a functional impairment in both rod- and cone- mediated vision has been extensively demonstrated in early/intermediate AMD eyes ${ }^{49-51}$.

We add to the literature by reporting a analysis of the photoreceptor damage in intermediate AMD eyes. Importantly, we quantified photoreceptor alteration by separately investigating regions with and without drusen. In order to provide an objective quantification of this damage, we measured the normalized reflectivity of the en face structural OCT image segmented at the level of the EZ. This assessment is not without challenges, including the presence of several factors that might impact on the OCT brightness and confound comparisons among subjects. This obstacle was resolved by "normalizing" the images-a technique that has been successfully employed in several prior studies, including in intermediate AMD eyes ${ }^{16,29,34-36}$.

Assessment of the EZ reflectivity has been used to investigate photoreceptor structure in different disorders, including macular telangiectasia (MacTel) type $2^{52}$, macular hole ${ }^{53,54}$, and $\mathrm{AMD}^{16,28,55}$. Pappuru et al. ${ }^{28}$ demonstrated that a lower EZ reflectivity is associated with worse visual acuities in eyes with intermediate AMD. In addition, the EZ reflectivity was demonstrated to be positively associated with ganglion cell loss, this supporting the postreceptoral hypothesis as rationale for the inner retinal impairment occurring in intermediate AMD eyes $^{16}$. Noteworthy, there is uncertain on the presence of ganglion cell loss in early/intermediate AMD eyes, as previous histologic findings suggest a loss in ganglion cells only in long-standing advanced $\mathrm{AMD}^{56}$, Furthermore, one previous study reported on the correlation between EZ integrity and retinal sensitivity in AMD eyes ${ }^{57}$. The authors of the latter study proved that retinal sensitivity significantly correlated with the status of the EZ in both early and late stages of AMD. Thus, the functional significance of the EZ in AMD eyes would seem to be adequately established.

We confirmed that photoreceptors are affected in intermediate AMD eyes. Importantly, we provided a topographical analysis of the normalized EZ reflectivity. Importantly, when we compared the normalized reflectivity of drusen-free areas to control eyes, it was significantly reduced, indicating that the photoreceptors localized in the regions without drusen also are abnormal in AMD patients. These results seem to provide imaging evidence to support the observations that photoreceptors are extensively affected in intermediate AMD eyes.

Although OCTA has significantly improved our capability to investigate the CC, many challenges must be addressed in analyzing the CC vasculature. In order to quantify the CC flow signal, en face OCTA images of this vascular layer may be binarized to separate pixels constituting these images into two groups: one that represents regions of flow signal and another representing signal deficits. Although a global threshold may be used to obtain binarized images of the CC, this approach may be problematic in presence of drusen that may cause local changes in image brightness that may be erroneously identified as FDs. In order to mitigate this issue, we employed an algorithm to compensate for the signal attenuation in the CC and we adopted a local threshold (Phansalkar method) which uses a small window rather than the whole image to determine the threshold for binarization. A study by Chu and colleagues ${ }^{44}$ has recently assessed the proper use of this threshold for CC binarization. In details, they demonstrated that applying a window radius of 2-4 pixels is the best strategy for analyzing $6 \times 6-\mathrm{mm}$ scans with a $1024 \times 1024$ size. Assuming this, we applied a window radius of 3 pixels $\left(\sim 103 \mu \mathrm{m}^{2}\right)$.

In recent years, OCTA technology has granted a considerable resource to study AMD. Several previous OCTA studies have described CC alterations in early and intermediate AMD eyes ${ }^{20-23,43,58,59}$. In agreement with these studies, we confirmed that intermediate AMD eyes are characterized by CC impairment. 
One of the most notable observations from our study was that the reduction in normalized EZ reflectivity is associated with CC flow signal in the "drusen-free" areas. As stated above, we chose to perform a separate analysis in the regions without drusen in order to investigate the relationship between CC flow signal and photoreceptors after restricting confounding factors (see the "Introduction" section for further details). Of note, our analysis was not able to incorporate the function of the outer blood-retina barrier, as we provided a rough correlation between the CC flow signal and photoreceptor structure. The relationship between these two structures was displayed in intermediate AMD eyes, while the absence of this association in healthy subjects may recommend the presence of a pathological dysregulation in the AMD group. These findings provided further evidence that photoreceptors and choriocapillaris are elements of a symbiotic unit in which dysfunctions of their components are strictly correlated.

Our study has limitations including the absence of a longitudinal evaluation. Another limitation of our image analysis is that media opacities and other causes of signal attenuation may have artifactiously caused a simultaneous reduced EZ reflectivity and CC flow signal, this producing a false positive correlation between these two structures. However, our algorithms were aimed at reducing this effect, as follows: (i) angiographic CC images were compensated for the signal attenuation using structural information, and (ii) the normalized EZ reflectivity was obtained with a process which compensates for the signal attenuation by using the RNFL and vitreous reflectivity. Furthermore, en face structural OCT and OCTA images must be interpreted with caution owing to a variety of image artifacts in the presence of drusen ${ }^{60}$. Thus, we felt the safer strategy was to perform an additional analysis limited to the "drusen-free" region. However, it must be noted that also drusen-free regions may be characterized by subtle changes as histopathology demonstrated the presence of basal linear deposits in areas without drusen ${ }^{61}$. Furthermore, the quantification of EZ reflectivity must be interpreted with caution as the OCT beam directionality may significantly influence this assessment ${ }^{62}$. Future studies will clarify if this aspect may have influenced our findings. Finally, we failed to provide a sophisticated topographical analysis as we only separately analyzed the regions without drusen. However, this is the first study to use imaging to directly correlate CC flow signal with photoreceptor damage in intermediate AMD eyes. We believe that the findings in our paper will be of help in further hypothesis generation and future study design.

In summary, in this SS-OCT structural and angiography study of the photoreceptors and CC, we observed that eyes with intermediate AMD have a diffuse reduced normalized EZ reflectivity. Importantly, the reduction in CC flow signal seems to be strongly associated with photoreceptor damage in areas without drusen. Future studies with longitudinal follow up may provide further insight into the interrelationship between photoreceptors, the choriocapillaris, and the pathogenesis of AMD.

\section{Data availability}

The data used to support the findings of this study are available from the corresponding author upon request.

Received: 14 May 2020; Accepted: 23 November 2020

Published online: 03 December 2020

\section{References}

1. Friedman, D. S. et al. Prevalence of age-related macular degeneration in the United States. Arch. Ophthalmol. 122, 564-572 (2004).

2. Ferris, F. L. et al. Clinical classification of age-related macular degeneration. Ophthalmology 120, 844-851 (2013).

3. Klein, R. et al. Markers of inflammation, oxidative stress, and endothelial dysfunction and the 20 -year cumulative incidence of early age-related macular degeneration. JAMA Ophthalmol. 132, 446 (2014).

4. Saksens, N. T. M. et al. Rare genetic variants associated with development of age-related macular degeneration. JAMA Ophthalmol. 134, 287 (2016).

5. Zarbin, M. A. \& Rosenfeld, P. J. Pathway-based therapies for age-related macular degeneration: An integrated survey of emerging treatment alternatives. Retina 30, 1350-1367 (2010).

6. Querques, G. et al. Treatment of dry age-related macular degeneration. Ophthalmic Res. 52, 107-115 (2014).

7. Sacconi, R. et al. Optical coherence tomography angiography in geographic atrophy. Retina https://doi.org/10.1097/IAE.00000 00000001873 (2017).

8. Bhutto, I. \& Lutty, G. Understanding age-related macular degeneration (AMD): Relationships between the photoreceptor/retinal pigment epithelium/Bruch's membrane/choriocapillaris complex. Mol. Aspects Med. 33, 295-317 (2012).

9. Campochiaro, P. A. Molecular pathogenesis of retinal and choroidal vascular diseases. Prog. Retinal Eye Res. 49, 67-81 (2015).

10. Saint-Geniez, M., Kurihara, T., Sekiyama, E., Maldonado, A. E. \& D’Amore, P. A. An essential role for RPE-derived soluble VEGF in the maintenance of the choriocapillaris. Proc. Natl. Acad. Sci. 106, 18751-18756 (2009).

11. Seddon, J. M. et al. Histopathological insights into choroidal vascular loss in clinically documented cases of age-related macular degeneration. JAMA Ophthalmol. https://doi.org/10.1001/jamaophthalmol.2016.3519 (2016).

12. Roberts, K. F. et al. Peripapillary choroidal thickness in healthy controls and patients with focal, diffuse, and sclerotic glaucomatous optic disc damage. Arch. Ophthalmol. 130, 980-986 (2012).

13. Mullins, R. F. et al. The membrane attack complex in aging human choriocapillaris: Relationship to macular degeneration and choroidal thinning. Am. J. Pathol. https://doi.org/10.1016/j.ajpath.2014.07.017 (2014).

14. Curcio, C. A., Millican, C. L., Allen, K. A. \& Kalina, R. E. Aging of the human photoreceptor mosaic: Evidence for selective vulnerability of rods in central retina. Investig. Ophthalmol. Vis. Sci. 34(12), 3278-3296 (1993).

15. Curcio, C. A., Medeiros, N. E. \& Millican, C. L. Photoreceptor loss in age-related macular degeneration. Investig. Ophthalmol. Vis. Sci. 37(7), 1236-1249 (1996).

16. Borrelli, E. et al. Postreceptor neuronal loss in intermediate age-related macular degeneration. Am. J. Ophthalmol. 181, 1-11 (2017).

17. Ramrattan, R. S. et al. Morphometric analysis of Bruch's membrane, the choriocapillaris, and the choroid in aging. Investig. Ophthalmol. Vis. Sci. 35(6), 2857-2864 (1994).

18. Sacconi, R. et al. Quantitative changes in the ageing choriocapillaris as measured by swept source optical coherence tomography angiography. Br. J. Ophthalmol. https://doi.org/10.1136/bjophthalmol-2018-313004 (2018).

19. Nassisi, M. et al. Topographic distribution of choriocapillaris flow deficits in healthy eyes. PLoS ONE 13, e0207638 (2018).

20. Borrelli, E., Sarraf, D., Freund, K. B. \& Sadda, S. R. OCT angiography and evaluation of the choroid and choroidal vascular disorders. Prog. Retin. Eye Res. https://doi.org/10.1016/j.preteyeres.2018.07.002 (2018). 
21. Borrelli, E., Uji, A., Sarraf, D. \& Sadda, S. R. Alterations in the choriocapillaris in intermediate age-related macular degeneration. Investig. Opthalmology Vis. Sci. 58, 4792-4798 (2017).

22. Borrelli, E. et al. Topographical analysis of the choriocapillaris in intermediate age-related macular degeneration. Am. J. Ophthalmol. 196, 34-43 (2018).

23. Borrelli, E. et al. Impact of choriocapillaris flow on multifocal electroretinography in intermediate age-related macular degeneration eyes. Investig. Ophthalmol. Vis. Sci. https://doi.org/10.1167/iovs.18-23943 (2018).

24. Braun, P. X. et al. Global analysis of macular choriocapillaris perfusion in dry age-related macular degeneration using swept-source optical coherence tomography angiography. Investig. Ophthalmol. Vis. Sci. https://doi.org/10.1167/iovs.19-27861 (2019).

25. Tiosano, L., Byon, I., Alagorie, A. R., Ji, Y. S. \& Sadda, S. R. Choriocapillaris flow deficit associated with intraretinal hyperreflective foci in intermediate age-related macular degeneration. Graefes Arch. Clin. Exp. Ophthalmol. https://doi.org/10.1007/s0041 7-020-04837-y (2020).

26. Staurenghi, G., Sadda, S., Chakravarthy, U., Spaide, R. F. \& International Nomenclature for Optical Coherence Tomography (IN OCT) Panel. Proposed lexicon for anatomic landmarks in normal posterior segment spectral-domain optical coherence tomography: The IN•OCT consensus. Ophthalmology 121, 1572-1578 (2014).

27. Hood, D. C. et al. The inner segment/outer segment border seen on optical coherence tomography is less intense in patients with diminished cone function. Investig. Opthalmol. Vis. Sci. 52, 9703 (2011).

28. Pappuru, R. R. et al. Relationship between outer retinal thickness substructures and visual acuity in eyes with dry age-related macular degeneration. Investig. Ophthalmol. Vis. Sci. 52, 6743-6748 (2011).

29. Borrelli, E., Palmieri, M., Viggiano, P., Ferro, G. \& Mastropasqua, R. Photoreceptor damage in diabetic choroidopathy. Retina https ://doi.org/10.1097/IAE.0000000000002538 (2019).

30. Querques, G. et al. Impact of reticular pseudodrusen on macular function. Retina 34, 321-329 (2014).

31. Carnevali, A. et al. Optical coherence tomography angiography: A useful tool for diagnosis of treatment-naïve quiescent choroidal neovascularization. Am. J. Ophthalmol. 169, 189-198 (2016).

32. Spaide, R. F. Choriocapillaris flow features follow a power law distribution: Implications for characterization and mechanisms of disease progression. Am. J. Ophthalmol. 170, 58-67 (2016).

33. Borrelli, E. et al. Rotational three-dimensional OCTA: A notable new imaging tool to characterize type 3 macular neovascularization. Sci. Rep. https://doi.org/10.1038/s41598-019-53307-x (2019).

34. Lee, S. Y., Stetson, P. F., Ruiz-Garcia, H., Heussen, F. M. \& Sadda, S. R. V. R. Automated characterization of pigment epithelial detachment by optical coherence tomography. Investig. Ophthalmol. Vis. Sci. 53, 164-170 (2012).

35. Charafeddin, W., Nittala, M. G., Oregon, A. \& Sadda, S. R. Relationship between subretinal hyperreflective material reflectivity and volume in patients with neovascular age-related macular degeneration following anti-vascular endothelial growth factor treatment. Ophthalmic Surg. Lasers Imaging Retina 46, 523-530 (2015).

36. Hu, Z., Nittala, M. G. \& Sadda, S. R. Comparison of retinal layer intensity profiles from different OCT devices. Ophthalmic Surg. Lasers Imaging Retina 44, S5-10 (2013).

37. Byon, I., Nassisi, M., Borrelli, E. \& Sadda, S. R. Impact of slab selection on quantification of choriocapillaris flow deficits by optical coherence tomography angiography. Am. J. Ophthalmol. https://doi.org/10.1016/j.ajo.2019.08.026 (2019).

38. Maruko, I., Kawano, T., Arakawa, H., Hasegawa, T. \& Iida, T. Visualizing large choroidal blood flow by subtraction of the choriocapillaris projection artifacts in swept source optical coherence tomography angiography in normal eyes. Sci. Rep. 8, 15694 (2018).

39. Zhang, Q. et al. A novel strategy for quantifying choriocapillaris flow voids using swept-source OCT angiography. Investig. Opthalmol. Vis. Sci. 59, 203-211 (2018).

40. Zhang, Q. et al. Projection artifact removal improves visualization and quantitation of macular neovascularization imaged by optical coherence tomography angiography. Ophthalmol. Retina. https://doi.org/10.1016/j.oret.2016.08.005 (2017).

41. Uji, A. et al. Choriocapillaris imaging using multiple en face optical coherence tomography angiography image averaging. JAMA Ophthalmol. https://doi.org/10.1001/jamaophthalmol.2017.3904 (2017).

42. Spaide, R. F. Choriocapillaris signal voids in maternally inherited diabetes and deafness and in pseudoxanthoma elasticum. Retina https://doi.org/10.1097/IAE.0000000000001497 (2017).

43. Borrelli, E. et al. Reduced choriocapillaris flow in eyes with type 3 neovascularization due to age-related macular degeneration. Retina https://doi.org/10.1097/IAE.0000000000002198 (2018).

44. Chu, Z. et al. Quantification of choriocapillaris with Phansalkar's local thresholding: Pitfalls to avoid. Am. J. Ophthalmol. https:// doi.org/10.1016/j.ajo.2020.02.003 (2020).

45. Zhang, Q. et al. Accurate estimation of choriocapillaris flow deficits beyond normal intercapillary spacing with swept source OCT angiography. Quant. Imaging Med. Surg. https://doi.org/10.21037/qims.2018.08.10 (2018).

46. Chen, L. et al. Nonexudative macular neovascularization supporting outer retina in age-related macular degeneration: A clinicopathologic correlation. Ophthalmology https://doi.org/10.1016/j.ophtha.2020.01.040 (2020).

47. Boretsky, A. et al. In vivo imaging of photoreceptor disruption associated with age-related macular degeneration: A pilot study. Lasers Surg. Med. https://doi.org/10.1002/1sm.22070 (2012).

48. Rogala, J. et al. In vivo quantification of retinal changes associated with drusen in age-related macular degeneration. Investig. Ophthalmol. Vis. Sci. 56, 1689-1700 (2015).

49. Feigl, B., Brown, B., Lovie-Kitchin, J. \& Swann, P. Cone- and rod- mediated multifocal eletroretinogram in early age-related maculopathy. Eye https://doi.org/10.1038/s.eye.6701503 (2005).

50. Owsley, C. et al. Psychophysical evidence for rod vulnerability in age-related macular degeneration. Investig. Ophthalmol. Vis. Sci. 41(1), 267-273 (2000).

51. Brown, B., Tobin, C., Roche, N. \& Wolanowski, A. Cone adaptation in age-related maculopathy. Optom. Vis. Sci. https://doi. org/10.1097/00006324-198606000-00009 (1986).

52. Sallo, F. B. et al. "En face" OCT imaging of the IS/OS junction line in type 2 idiopathic macular telangiectasia. Investig. Opthalmol. Vis. Sci. 53, 6145 (2012).

53. Oh, J., Smiddy, W. E., Flynn, H. W., Gregori, G. \& Lujan, B. Photoreceptor inner/outer segment defect imaging by spectral domain OCT and visual prognosis after macular hole surgery. Investig. Opthalmol. Vis. Sci. 51, 1651 (2010).

54. Villate, N., Lee, J. E., Venkatraman, A. \& Smiddy, W. E. Photoreceptor layer features in eyes with closed macular holes: Optical coherence tomography findings and correlation with visual outcomes. Am. J. Ophthalmol. 139, 280-289 (2005).

55. Takahashi, A. et al. Photoreceptor damage and reduction of retinal sensitivity surrounding geographic atrophy in age-related macular degeneration. Am. J. Ophthalmol. 168, 260-268 (2016).

56. Medeiros, N. E. \& Curcio, C. A. Preservation of ganglion cell layer neurons in age-related macular degeneration. Investig. Ophthalmol. Vis. Sci. 42(3), 795-803 (2001).

57. Landa, G., Su, E., Garcia, P. M. T., Seiple, W. H. \& Rosen, R. B. Inner segment-outer segment junctional layer integrity and corresponding retinal sensitivity in dry and wet forms of age-related macular degeneration. Retina 31, 364-370 (2011).

58. Vujosevic, S. et al. Quantitative choriocapillaris evaluation in intermediate age-related macular degeneration by swept-source optical coherence tomography angiography. Acta Ophthalmol. https://doi.org/10.1111/aos.14088 (2019).

59. Cicinelli, M. V. et al. Choroid morphometric analysis in non-neovascular age-related macular degeneration by means of optical coherence tomography angiography. Br. J. Ophthalmol. https://doi.org/10.1136/bjophthalmol-2016-309481 (2017). 
60. Lane, M. et al. Visualizing the choriocapillaris under drusen: Comparing 1050-nm swept-source versus 840-nm spectral-domain optical coherence tomography angiography. Investig. Opthalmology Vis. Sci. 57, OCT585 (2016).

61. Chen, L. et al. Abundance and multimodal visibility of soft drusen in early age-related macular degeneration: A clinicopathologic correlation. Retina https://doi.org/10.1097/IAE.0000000000002893 (2020).

62. Gao, W., Cense, B., Zhang, Y., Jonnal, R. S. \& Miller, D. T. Measuring retinal contributions to the optical Stiles-Crawford effect with optical coherence tomography. Opt. Express https://doi.org/10.1364/oe.16.006486 (2008).

\title{
Acknowledgements
}

The PLEX Elite 9000 has been made available through the Advanced Retina Imaging Network for which Giuseppe Querques is a steering Committee Member.

\section{Author contributions}

E.B. Study supervision. Concept and study design. Data collection. Data interpretation. Data analysis and statistics. Drafting, revision and final approval of manuscript. B.Z., M.C., A.B. Data collection. R.S., I.Z., L.Q. Data collection. Revision and final approval of manuscript. F.B., G.Q. Study supervision. Concept and study design. Data interpretation. Revision and final approval of manuscript.

\section{Competing interests}

The authors declare that there are no conflicts of interest regarding the publication of this article. Francesco Bandello is a consultant for: Alcon (Fort Worth,Texas,USA), Alimera Sciences (Alpharetta, Georgia, USA), Allergan Inc (Irvine, California,USA), Farmila-Thea (Clermont-Ferrand, France), Bayer Shering-Pharma (Berlin, Germany), Bausch And Lomb (Rochester, New York, USA), Genentech (San Francisco, California, USA), HoffmannLa-Roche (Basel, Switzerland), Novagali Pharma (Évry, France), Novartis (Basel, Switzerland), Sanofi-Aventis (Paris, France), Thrombogenics (Heverlee,Belgium), Zeiss (Dublin, USA). Giuseppe Querques is a consultant for: Alimera Sciences (Alpharetta, Georgia, USA), Allergan Inc (Irvine, California, USA), Amgen (Thousand Oaks ,USA), Bayer Shering-Pharma (Berlin, Germany), Heidelberg (Germany), KBH (Chengdu; China), LEH Pharma (London, UK), Lumithera (Poulsbo; USA), Novartis (Basel, Switzerland), Sandoz (Berlin, Germany), Sifi (Catania, Italy), Sooft-Fidea (Abano, Italy), Zeiss (Dublin, USA). The other authors have no disclosures.

\section{Additional information}

Correspondence and requests for materials should be addressed to G.Q.

Reprints and permissions information is available at www.nature.com/reprints.

Publisher's note Springer Nature remains neutral with regard to jurisdictional claims in published maps and institutional affiliations.

\begin{abstract}
Open Access This article is licensed under a Creative Commons Attribution 4.0 International License, which permits use, sharing, adaptation, distribution and reproduction in any medium or format, as long as you give appropriate credit to the original author(s) and the source, provide a link to the Creative Commons licence, and indicate if changes were made. The images or other third party material in this article are included in the article's Creative Commons licence, unless indicated otherwise in a credit line to the material. If material is not included in the article's Creative Commons licence and your intended use is not permitted by statutory regulation or exceeds the permitted use, you will need to obtain permission directly from the copyright holder. To view a copy of this licence, visit http://creativecommons.org/licenses/by/4.0/.
\end{abstract}

(C) The Author(s) 2020 